\title{
Upper eyelid platinum weight placement for the treatment of paralytic lagophthalmos: A new plane between the inner septum and the levator aponeurosis
}

\author{
Tae Suk Oh, Kyunghyun Min, Sin Young Song, Jong Woo Choi, Kyung Suk Koh \\ Department of Plastic and Reconstructive Surgery, Asan Medical Center, University of Ulsan College of Medicine, Seoul, Korea
}

Background The most common surgical treatment for paralytic lagophthalmos is the placement of a weight implant in the upper eyelid; however, this technique confers the risks of implant visibility, implant extrusion, and entropion. In this study, we present a new technique of placing platinum weight implants between the levator aponeurosis and inner septum to decrease such complications.

Methods A total of 37 patients with paralytic lagophthalmos were treated between March 2014 and January 2017 with platinum weight placement (mean follow-up, 520.1 days). After dissecting through the orbicularis oculi muscle, the tarsal plate and levator aponeurosis were exposed. The platinum weights (1.0-1.4 g) were fixed to the upper margin of the tarsal plate and placed underneath the orbital septum.

Results Five patients could partially close their eye after surgery. The average distance between the upper eyelid and the lower eyelid when the eyes were closed was $1.12 \mathrm{~mm}$. The rest of the patients were able to close their eye completely. Three patients patient developed allergic conjunctivitis after platinum weight insertion, which was managed with medication. None of the patients complained of discomfort in the upper eyelid after surgery. Visibility or extrusion of the implant were observed in three patients.

Conclusions Postseptal weight placement is a safe and reproducible method in both primary and secondary upper eyelid surgery for patients with paralytic lagophthalmos. It is a feasible method for preventing implant visibility, implant exposure, and entropion. Moreover, platinum is a better implant material than gold because of its smaller size and greater thinness.

Keywords Platinum / Prostheses and implants / Eyelid diseases / Facial paralysis
Correspondence: Tae Suk Oh Department of Plastic and Reconstructive Surgery, Asan Medical Center, University of Ulsan College of Medicine, 88 Olympic-ro 43-gil, Songpa-gu, Seoul 05505, Korea Tel: $+82-2-3010-3607$

Fax: +82-2-476-7471

E-mail: tasuko@amc.seoul.kr

The article was presented at the PRS Korea 2015 on November 13-15, 2015 in Seoul, Korea.

\section{INTRODUCTION}

Lagophthalmos is a condition characterized by prolonged exposure of the eyeball due to paralysis of the orbicularis oculi mus- cle, thyroid disease, exophthalmos caused by an orbital tumor, or traumatic eyelid injury [1]. Persistent exposure of the cornea can evolve into exposure keratitis or conjunctivitis. In addition, it can also lead to the development of corneal ulcer, corneal per- 
foration, and even blindness [2]. Consequently, many methods for protecting the cornea have been suggested. Conservative nonsurgical treatment includes the use of artificial tears, ointments, and therapeutic contact lenses [3]. However, if nonsurgical approaches are not effective, lagophthalmos should be corrected using surgical methods.

The most commonly used method based on the insertion plane is pretarsal insertion. By using a transcutaneous approach, the weight is placed into the pretarsal space. The pocket is deep to the orbicularis oculi muscle. Then, skin is redraped over the weight implant for eye closure. However, there is a potential risk of implant visibility and extrusion. Rozen and Lehrman [1] suggested a postseptal insertion position to overcome these drawbacks. In that approach, the septum is incised 2 to $3 \mathrm{~mm}$ above the superior border of the tarsus, thus leaving a septal margin for closure after weight placement. The postseptal fat is retracted superiorly, the underlying levator aponeurosis is identified, and further dissection superiorly reveals the red levator muscle fibers. The pocket is dissected 2 to $3 \mathrm{~mm}$ medial to the medial limbus.

The most common surgical treatment for lagophthalmos is the insertion of a gold weight implant in the upper eyelid. This

\section{Fig. 1. Gold and platinum weights}

The density of platinum at room temperature is $21.45 \mathrm{~g} / \mathrm{cm}^{3}, \mathrm{com}-$ pared with $19.3 \mathrm{~g} / \mathrm{cm}^{3}$ for gold. Therefore, if the same mass is needed, the volume of platinum can be reduced by $>10 \%$ compared with gold.

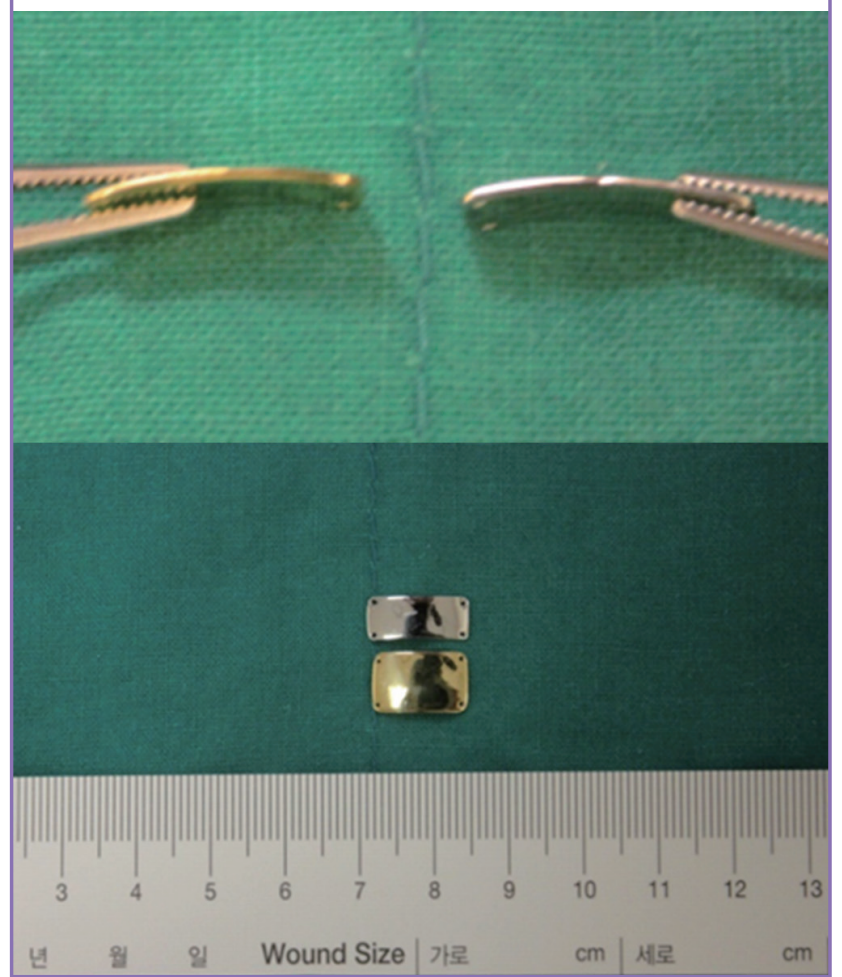

technique enables eyelid closing, which can protect the cornea. It does not have any harmful effects on the visual field or visual acuity. Moreover, the operation, revision, and removal of the gold weight are relatively simple.

However, gold weight insertion has some pitfalls. First, implant visibility can occur because the skin of the eyelid is thin. Second, platinum is denser at room temperature $\left(21.45 \mathrm{~g} / \mathrm{cm}^{3}\right)$ than gold $\left(19.3 \mathrm{~g} / \mathrm{cm}^{3}\right)$ (Fig. 1). This means that a gold weight implant needs more space for its insertion inside the eyelid, resulting in inevitable bulkiness. Lastly, capsule formation requiring subsequent implant removal is possible. Therefore, platinum is a better choice than gold. Nevertheless, the problems of visibility and bulkiness remain [3]. Although many physicians have suggested various implant insertion planes to minimize these problems, no consensus has been reached.

In this study, we present a feasible and efficacious technique with a potentially decreased risk of complications because the weight implant is inserted into a deeper space in the upper eyelid.

\section{METHODS}

A total of 37 patients with lagophthalmos treated from March 2014 to January 2017 were reviewed retrospectively with Institutional Review Board approval (IRB No. 2018-0170). Subjects received informed consent for photography. All patients underwent eyelid weight placement in the plastic and reconstructive surgery department of our center. The male-to-female ratio was $20: 17$, and the mean patient age was 48.3 years (range, $12-80$ years). The most common cause of lagophthalmos was postoperative facial palsy after tumor resection $(n=20)$. The mean weight of the eyelid implant was $1.188 \mathrm{~g}$ (range, 1.0-1.4 g). The mean size of the platinum weight was $13.9 \times 5.9 \times 1.18 \mathrm{~mm}^{3}$. The mean follow-up period was 520.1 days (range, 105-708 days) (Table 1).

\begin{tabular}{l}
$\begin{array}{l}\text { Table } 1 . \text { Demographic and clinical } \\
\text { patients }(\mathbf{n = 3 7})\end{array}$ \\
\begin{tabular}{|lc|}
\hline Characteristic & Value \\
\hline Sex (male:female) & $20: 17$ \\
Age (yr) & $48.3(12-80)$ \\
Weight (g) & $1.188(1.0-1.4)$ \\
Mean platinum weight size (mm) & $13.9 \times 5.9 \times 1.18$ \\
Follow-up (day) & $520.1(105-708)$ \\
Incomplete closure & $5(13.5)$ \\
Extrusion & $2(5.4)$ \\
Plate visibility & $1(2.7)$ \\
Allergic conjunctivitis & $3(8.1)$ \\
\hline Values are presented as mean (range) or number (\%).
\end{tabular} \\
\hline
\end{tabular}




\section{Fig. 2. Postseptal platinum weight placement}

We hypothesized that postseptal insertion, in contrast to pretarsal insertion (A), would rarely cause implant visibility and extrusion because the fat tissue can cover the platinum implant properly (B).

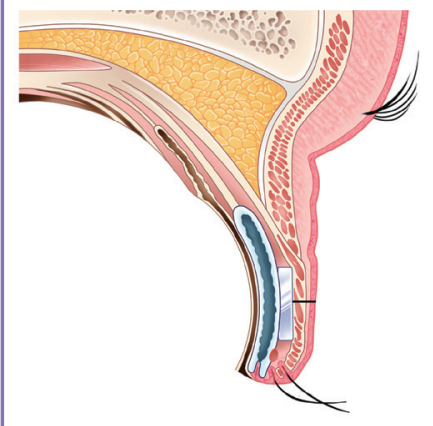

A

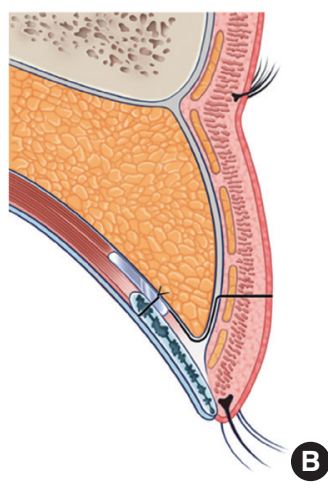

\section{Fig. 3. Insertion plane of the platinum weight}

Dissection was performed to separate the levator aponeurosis and to expose the tarsal plate. The platinum weight was then inserted into the space between the levator aponeurosis and the orbital inner septum.

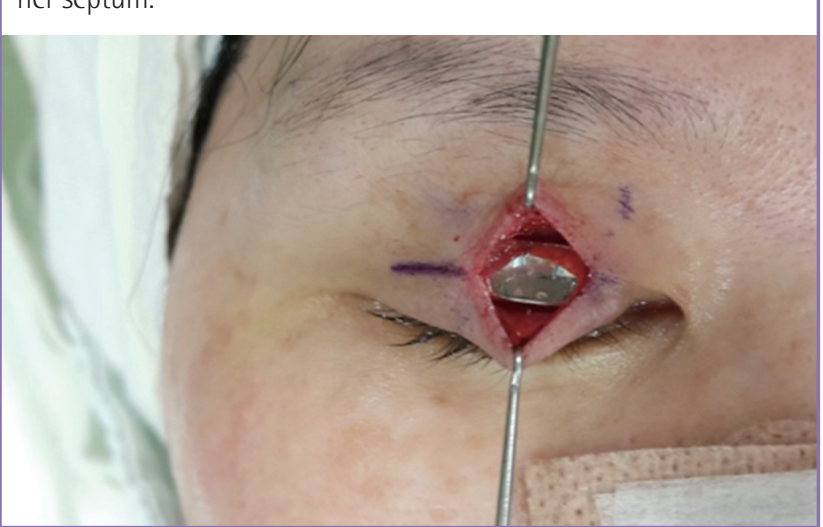

Fig. 4. Platinum weight insertion: case 1 (lateral view)

A 55-year-old female patient underwent brain tumor surgery and experienced left facial palsy, resulting in lagophthalmos. (A) Preoperative lateral view. (B) Fifteen-month postoperative lateral view. No visible protrusion was noted.

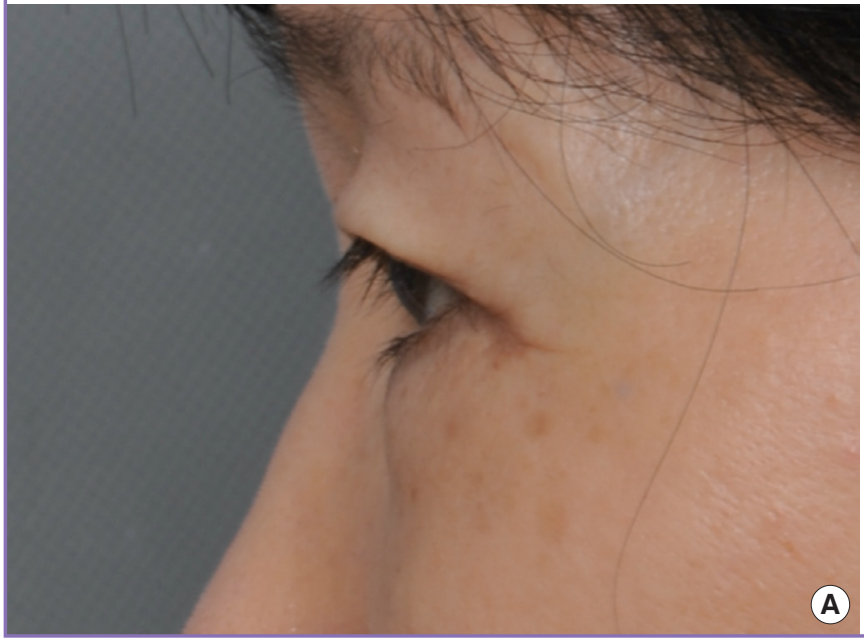

\section{Surgical technique}

All operations were performed as outpatient procedures. The preoperative skin incision design was made at the pretarsal skin crease. To determine the implant weight, the estimated gold (or platinum) weight was attached to the eyelid with tiny adhesive tape. The patients opened and closed their eye. The operator checked the symmetry of both eyes and finally selected the exact weight of the implant. With the patient in the supine position, skin draping was done with $0.5 \%$ chlorhexidine. The incision was made with a No. 15 blade. Dissection was performed to separate the levator aponeurosis and to expose the tarsal plate. Then, the platinum weight was inserted into the space between the levator aponeurosis and the orbital inner septum (Figs. 2, 3). Two-point suture fixation with nylon 6-0 was done

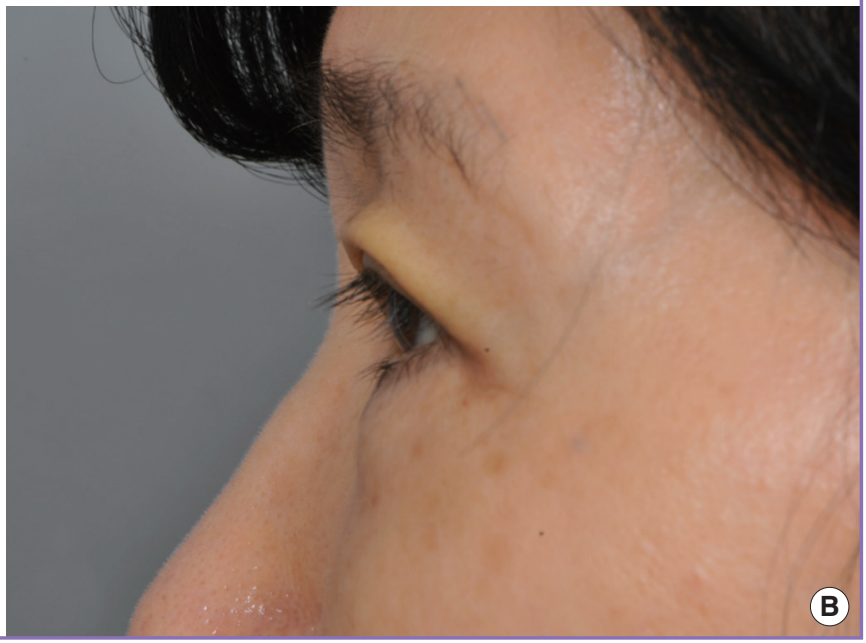

to the tarsus. A temporary skin suture was made using silk 6-0. The patient's position was changed to sitting, and the patient was asked to open, close, and blink his or her eyes for the evaluation of the immediate postoperative status. Final skin closure was performed with silk 6-0 sutures.

\section{Assessment of safety and effectiveness}

All cases of revision were analyzed to understand the safety and efficacy of the procedure. The rates of revision and implant removal because of complications were collected, and the percentage for each procedure was calculated.

For the evaluation of the effectiveness based on preoperative and postoperative measurements, the closure difference was measured at the outpatient clinic. 


\section{Fig. 5. Platinum weight insertion: case 1 (frontal view)}

Photographs were taken before surgery and at 15 months postoperatively. Eye closure was possible. (A) Preoperative frontal view. (B) Postoperative frontal view.
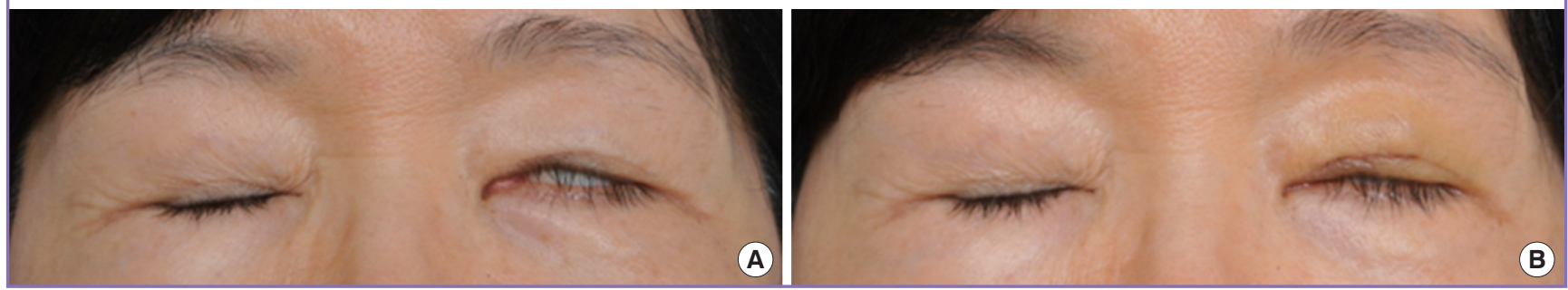

\section{Fig. 6. Platinum weight insertion: case 2}

$(A, B)$ A 30-year-old male presented with right facial palsy after facial fracture and underwent platinum weight insertion. (C, D) Full eye opening and closure were possible 12 months after the operation.
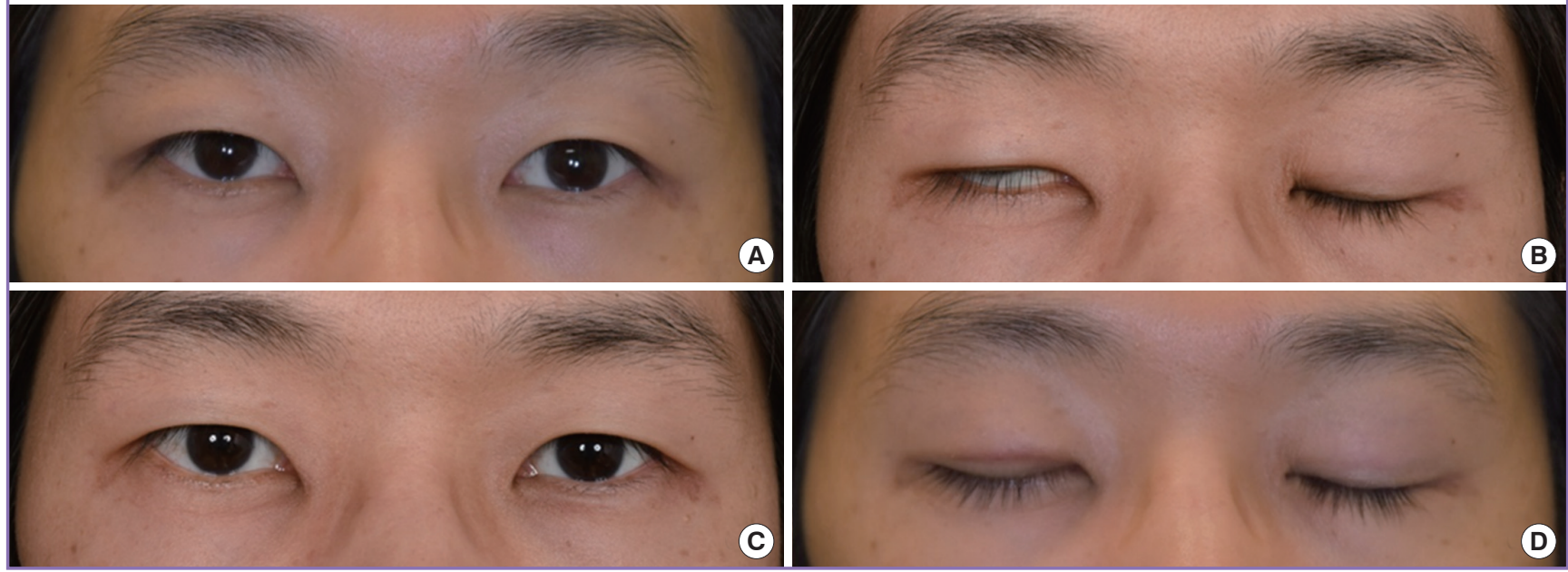

\section{RESULTS}

Of the 37 patients, 32 were able to close their eye completely after the surgery (Figs. 4-6). In patients who could not fully close their eye, the average distance between the upper eyelid and the lower eyelid when the eyes were closed was $1.12 \mathrm{~mm}$. Three patients developed allergic conjunctivitis after platinum weight insertion, which was managed with topical medication. Three patients underwent a revision procedure because of discomfort resulting from incomplete closure and extrusion. Only one patient complained of implant visibility. Another patient commented about an upper eyelid height asymmetry; however, this patient underwent upper blepharoplasty concomitantly with weight insertion.

\section{DISCUSSION}

In 1972, Levine reported that surgical procedures are required if nonsurgical methods are not effective for lagophthalmos. This triggered the rapid development of new surgical techniques, in- cluding tarsorrhaphy, temporalis muscle transfer, and weight insertion $[2,4,5]$. Tarsorrhaphy, known as the McLaughlin procedure [6], is a conventional method that preserves the cornea by suturing the superior and inferior tarsal plate of the epicanthus. However, this procedure narrows the visual field and results in difficulty in separating the lids. Temporalis muscle transfer is a technique in which the temporalis muscle is connected to the tarsal plate. This allows the eyelid to blink with mastication. However, it results in a bulky muscle volume and gradual decrease of function.

Gold plate insertion for the treatment of lagophthalmos was first reported in 1958 by Illig [7]. Thereafter, technical improvements have been reported. Berghaus et al. [8] suggested using a platinum chain instead of gold because the density of platinum makes it the most useful material for a metal implant in the upper eyelid.

The insertion plane of the gold or platinum weight is important not only for functional and aesthetic reasons, but also for reducing complications. The skin of the upper eyelid is thin enough to cause visibility or extrusion of the implant. Further- 
more, skin erosion and ulceration can occur. For these reasons, several studies have been published about dissection methods and anatomical considerations for insertion $[2,9,10]$. Most recently, Rozen and Lehrman [1] introduced the method of attaching platinum to the aponeurosis of the levator muscle. However, that method encountered the problem that the preoperatively estimated weight was not the same as the actual inserted weight.

In this study, we designed an approach of inserting a platinum weight between the levator aponeurosis and inner septum. We hypothesized that this approach, in contrast to previous approaches, would rarely cause implant visibility and extrusion because the preaponeurotic fat tissue could cover the platinum implant properly. Furthermore, the preoperatively measured platinum weight was directly inserted into the eyelid. Favorable results were obtained with this method.

All eyelids of the 37 patients could be closed postoperatively. However, five patients could close their eye only partially, and the average gap between the upper and lower eyelids was 1.12 $\mathrm{mm}$. A revision procedure was performed in three patients. The reasons for revision were extrusion ( 1 case) and incomplete eye closure ( 2 cases). Two patients underwent gold weight insertion at another hospital (Figs. 7, 8).
Only one case of reoperation (due to extrusion) was reported. This patient had undergone a previous insertion of a gold weight implant. None of the patients in whom a platinum weight was inserted developed extrusion. The results in the patients who received a platinum implant were similar to those reported in other studies $[3,11]$. Moreover, the platinum implants were smaller and thinner than the corresponding gold implants would have been, producing a more favorable aesthetic outcome.

No patient complained of severe irritation or corneal skin ulceration. Moreover, there were no cases of postoperative visual disturbance or astigmatism. In other studies, corneal skin ulceration and severe irritation of the eye were reported in up to $12.2 \%$ of patients $[1,9]$. We believe that the improved results in this study are attributable to the postseptal insertion method.

Bladen et al. [12] compared the cosmetic outcomes between gold weight and platinum weight implants. Patients in whom a gold weight was inserted experienced eyelid drooping (5/9, $55.6 \%)$ and flattening $(2 / 9,22.2 \%)$. However, among patients in whom a platinum chain was inserted, only two cases of mild eyelid prominence were recorded.

Because the platinum weight was inserted above the levator aponeurosis, we were concerned about functional eye closure.

\section{Fig. 7. Platinum weight insertion: case 3}

A 56-year-old female underwent a gold weight insertion due to left facial paralysis 18 years after a schwannoma operation. The position of that gold weight changed, causing visibility and discomfort. Removal and platinum weight insertion were performed. (A) The gold weight (1.3 g) was removed from the pretarsal space. (B) A capsule had formed at the insertion site, so capsulectomy was performed and a new platinum weight was inserted on the tarsal plate.
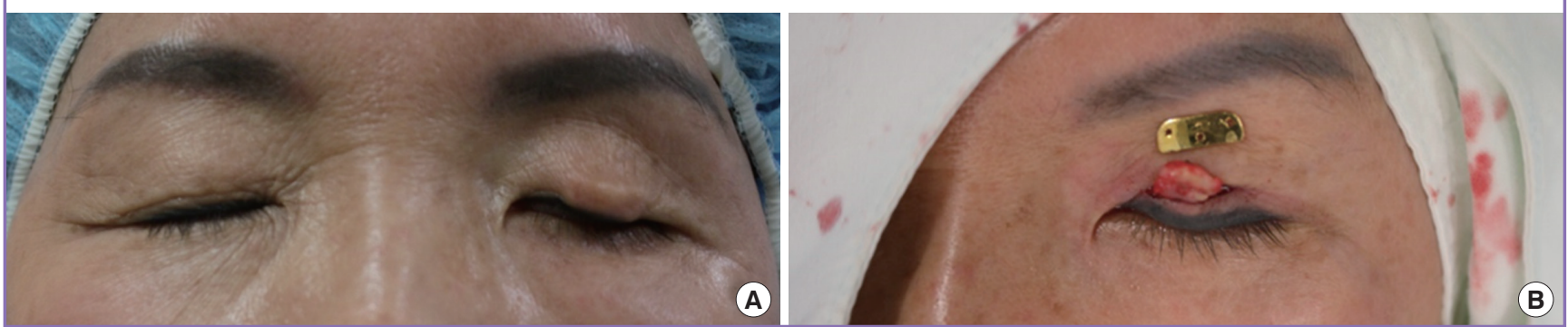

Fig. 8. Case 3 at 16 months postoperatively

$(A, B)$ The patient could completely close her left eye. Mild visibility was noted when the eyes were closed. However, the patient considered the results acceptable compared with the previous gold weight insertion.
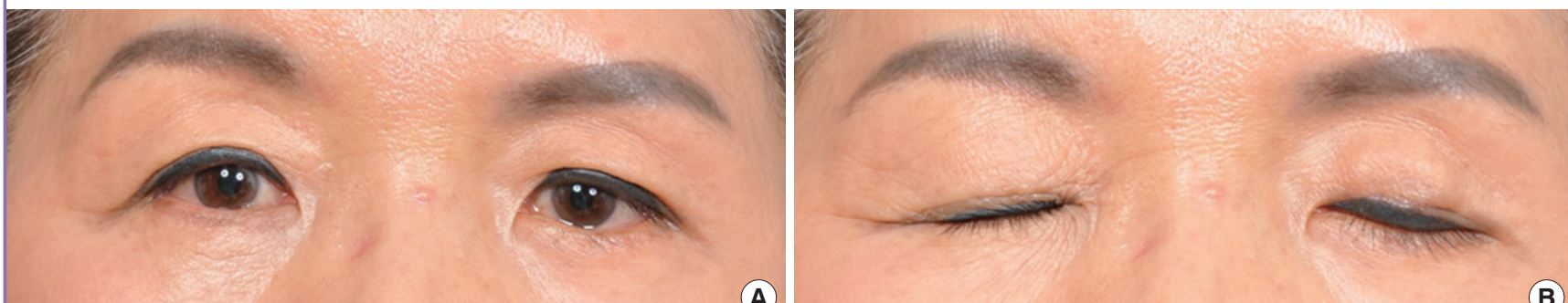
When the eyes open and close, the levator muscle glides; thus, the platinum weight can affect the gliding surface. However, no impairment of eye closure was observed, and the levator muscle function was intact. One patient who underwent gold weight insertion complained of extrusion. Therefore, the gold weight was removed and a platinum weight was inserted. As capsule formation was noted around the extracted gold implant, we believe that further investigation should be done concerning the association between upper eyelid movement and capsule formation.

In this study, a revision procedure was performed in three patients $(3 / 37,8.1 \%)$. This rate is lower than has been reported in previous studies (10.5\%, Wagh et al. [13]; 12.7\%, Tan et al. [5]). Silver et al. [3] reported a lower complication rate (5.9\%) than that in our study. They used a platinum weight with a thin profile for lagophthalmos correction. However, in their study, the visibility of the metal implant was not analyzed.

No previous studies discussed the effects of implant insertion during sleeping. One of the main problems in patients with lagophthalmos is improper eye closure during sleeping. This often causes eye dryness, discomfort, and shallow sleep. The mechanism of platinum weight insertion is based on gravitational pull on the eyelid; however, the effect of gravity is small in the supine position. This problem should be investigated further.

This study has some limitations. First, the total number of patients $(\mathrm{n}=37)$ was relatively small. Second, the outpatient clinic follow-up period was not long. Third, data on long-term complications, such as exacerbation of extrusion and capsule formation, were not collected.

An analysis of a larger group of patients would more precisely clarify the rates of success, complications, and revision of platinum weight implants. Furthermore, continuous follow-up would allow the evaluation of capsule formation around the platinum implants, as well as postoperative eye opening and closure. This would be helpful for selecting the weight of the platinum implant, reducing the revision and complication rates, and maximizing patient satisfaction.

In conclusion, fixation of a platinum weight implant in the superior tarsus, on the levator aponeurosis and underneath the orbital inner septum, is a compelling new technique for correcting lagophthalmos that markedly decreases the rates of implant extrusion and skin ulceration. This technique may be a new standard for determining the incision and dissection plane for platinum weight insertion. Furthermore, we are confident that with the accumulation of cases and long-term outpatient follow-up data, the superiority of postseptal platinum implant insertion can be proven.

\section{NOTES}

\section{Conflict of interest}

No potential conflict of interest relevant to this article was reported.

\section{Ethical approval}

The study was approved by the Institutional Review Board of Asan Medical Center (IRB No. 2018-0170) and performed in accordance with the principles of the Declaration of Helsinki. Written informed consents were obtained.

\section{Patient consent}

The patients provided written informed consent for the publication and the use of their images.

\section{REFERENCES}

1. Rozen S, Lehrman C. Upper eyelid postseptal weight placement for treatment of paralytic lagophthalmos. Plast Reconstr Surg 2013;131:1253-65.

2. Choi HY, Hong SE, Lew JM. Long-term comparison of a newly designed gold implant with the conventional implant in facial nerve paralysis. Plast Reconstr Surg 1999;104:162434.

3. Silver AL, Lindsay RW, Cheney ML, et al. Thin-profile platinum eyelid weighting: a superior option in the paralyzed eye. Plast Reconstr Surg 2009;123:1697-703.

4. Baheerathan N, Ethunandan M, Ilankovan V. Gold weight implants in the management of paralytic lagophthalmos. Int J Oral Maxillofac Surg 2009;38:632-6.

5. Tan ST, Staiano JJ, Itinteang T, et al. Gold weight implantation and lateral tarsorrhaphy for upper eyelid paralysis. J Craniomaxillofac Surg 2013;41:e49-53.

6. Mclaughlin CR. Surgical support in permanent facial paralysis. Plast Reconstr Surg (1946) 1953;11:302-14.

7. Illig KM. A new method of lagophthalmos surgery. Klin Monbl Augenheilkd Augenarztl Fortbild 1958;132:410-1.

8. Berghaus A, Neumann K, Schrom T. The platinum chain: a new upper-lid implant for facial palsy. Arch Facial Plast Surg 2003;5:166-70.

9. Amer TA, El-Minawi HM, El-Shazly MI. Low-level versus high-level placement of gold plates in the upper eyelid in patients with facial palsy. Clin Ophthalmol 2011;5:891-5.

10. Lessa S, Nanci M, Sebastia R, et al. Treatment of paralytic lagophthalmos with gold weight implants covered by levator aponeurosis. Ophthal Plast Reconstr Surg 2009;25:189-93.

11. Bianchi B, Ferri A, Leporati M, et al. Upper eyelid platinum 
chain placement for treating paralytic lagophthalmos. J Craniomaxillofac Surg 2014;42:2045-8.

12. Bladen JC, Norris JH, Malhotra R. Cosmetic comparison of gold weight and platinum chain insertion in primary upper eyelid loading for lagophthalmos. Ophthal Plast Reconstr
Surg 2012;28:171-5.

13. Wagh VK, Lim WS, Cascone NC, et al. Post-septal upper eyelid loading for treatment of exposure keratopathy secondary to non-cicatricial lagophthalmos. Orbit 2016;35: 239-44. 